\title{
Effect of Disulfide Bonds in Antifungal Peptide from Petunia Hybrida: A Molecular Dynamics Study
}

\author{
M. Jayanthi ${ }^{1}$, N.A. Udaya Prakash $^{2}$, P. Kangueane ${ }^{1,3}$, Z. A. Rafi ${ }^{4}$ and K. Sekar ${ }^{*, 2,5}$ \\ ${ }^{I}$ School of Biotechnology, Chemical and Biomedical Engineering, VIT University, Vellore 632 014, India \\ ${ }^{2}$ Bioinformatics Centre, (Centre of excellence in Structural Biology and Bio-computing), Indian Institute of Science, \\ Bangalore 560 012, India \\ ${ }^{3}$ Biomedical Informatics, 17 A, Irulan Sandy Annex, Pondicherry 607 402, India \\ ${ }^{4}$ Centre of Excellence in Bioinformatics, School of Biotechnology, Madurai Kamaraj University, Madurai 625 021, \\ India \\ ${ }^{5}$ Supercomputer Education and Research Centre, Indian Institute of Science, Bangalore 560 012, India
}

\begin{abstract}
The solution structure of a novel plant defensin (PhD1) contains a fifth disulfide bond, unlike other plant defensins, which have four disulfide bonds. The present study aims to better understand the stability, thermal dependence and the role of disulfide bonds in the tertiary structure of PhD1 using Molecular Dynamics (MD) simulations. The secondary structures are intact in the native structure simulated at $300 \mathrm{~K}$. However, in the mutant structures a small variation is observed. A significant shift in the peptide conformation is observed when the additional fifth disulfide bond (Cys7-Cys23) is mutated. No large change in the tertiary structure conformation is observed till $400 \mathrm{~K}$, which demonstrates the high thermal stability of the protein. Here, we also show that the mutation of disulfide bonds did not result in a drastic conformational change. The antifungal property along with high structural stability of the plant defensin protein makes it a promising candidate for the development of novel fungicides.
\end{abstract}

Keywords: Antifungal peptide, disulfide bond, temperature resistance, cysteine mutations and molecular dynamics simulations.

\section{INTRODUCTION}

Antifungal proteins are synthesized by plants to prevent invasion of fungi. It is well known that plants have no immune system and thus antifungal proteins are produced in either constitutive or induced manner [1]. Further, defenserelated proteins are distributed widely throughout the plant kingdom. They are highly divergent in their sequences and the mode of action of these peptides is still not clearly understood [2]. Among the vast array of antifungal peptides, the cysteine-rich peptides form a large group in which certain disulfide motif stabilizes the molecular scaffold. In addition, the cysteine rich peptides are small, highly basic and these are usually classified by the number and pairing pattern of the disulfide bonds [3, 4]. As stated in the literature, a series of three-dimensional structures belonging to this group of antifungal peptides including thionins [5], plant defensins [6, 7], hevein-like [8] and knottin-like [9] have been investigated and reported. Plant defensins are typically 45-54 amino acid residues long, containing eight cysteine residues that form four disulfide bonds and are characterized from various plant tissues including leaves,

*Address correspondence to this author at the Bioinformatics Centre, (Centre of excellence in Structural Biology and Bio-computing), Supercomputer Education and Research Centre, Indian Institute of Science, Bangalore 560 012, India; Tel: +91-80-22933059/22932469/23601409; Fax: +91-80-23600683/23600551; E-mail: sekar@serc.iisc.ernet.in and sekar@physics.iisc.ernet.in pods, tubers, fruits and flowers. Much of the work, however, has been performed on seeds where these proteins are prevalent [10]. All plant defensins share a characteristic three-dimensional folding pattern ( $\alpha \beta$-motif), stabilized by four disulfide bonds $[11,12]$.

A novel plant defensin (PhD1) with five disulfide bonds isolated from the flowers of Petunia hybrida [13] is composed of an $\alpha$-helix, three $\beta$-strands and two loop regions (L1 and L2). The loop L1 is found to connect $\alpha$-helix and $\beta_{1^{-}}$ strand and loop L2 connects $\beta_{2}$-strand with $\beta_{3}$-strand. The protein $\mathrm{PhD} 1$ has an extra cysteine pair (Cys7-Cys23) that forms the fifth disulfide bond. The characteristic feature of the protein $\mathrm{PhD} 1$ is its cystine knot formed by three disulfide bonds (Cys7-Cys23, Cys14-Cys34 and Cys20-Cys41) with one disulfide penetrating through a macrocycle formed by the two other disulfides and interconnecting peptide backbones $[14,15]$. It is not known whether plant defensins have a common mode of action [16]. However, Rs-AFP2, one of the best characterized antifungal plant defensins, has been suggested to interact with a membrane-bound receptor rather than by direct defensin-lipid interaction. Structurally, the residues essential for antifungal activity are located at the two adjacent patches on the surface of Rs-AFP2 [17]. The equivalent residues in the structure of PhD1 also form two patches with differences in electrostatic characteristics suggesting that the receptor for PhD1 may be different from the receptor of Rs-AFP2 [8]. The protein PhD1 inhibits the growth of phytopathogenic fungi, namely, Botrytis cinerea 
and Fusarium oxysporum f. sp. dianthi and may thus be of interest for the development of novel fungicides [18].

Molecular dynamics (MD) simulation studies have been used over the last 15 years to better understand the structure and the conformational change in proteins. Recent advances in the computing resources and improvements in accuracy of force fields used to describe biological macromolecules, have allowed the reproduction of experimental results in MD simulations [19]. These advances have created the possibility of direct comparison of MD calculations with experimental data. The available experimental data of floral defensin, $\mathrm{PhD} 1$ provide a good starting point for MD investigations. Study on the important properties of $\mathrm{PhDl}$ is an area of interest to better understand its mechanism of action and to develop strategies for applications in developing effective antimicrobial compounds. The present work aims to study the effect of different temperatures on the structure of the plant defensin protein PhD1 and a possible role of the cysteine residues involved in the disulfide bonds on the tertiary structure. The results of the molecular dynamics simulations are described in the subsequent sections.

\section{MATERIALS AND METHODOLOGY}

\subsection{Molecular Model}

The three-dimensional atomic coordinates of $\mathrm{PhD1}$, a cysteine-rich antifungal protein (Fig. 1) composed of 47 amino acid residues isolated from the flowers of the plant Petunia hybrida [PDB-Id: 1N4N] were downloaded from the locally maintained (Bioinformatics Centre, Indian Institute of Science, Bangalore, India) PDB-FTP server. The in silico mutants were generated using the program Swiss PDB viewer [20]. The cysteine residues at positions 3, 7, 14, 20 and 24 in the native structure [PDB-Id: $1 \mathrm{~N} 4 \mathrm{~N}$ ] were mutated to alanine residues and the five single mutants were named as C3A, C7A, C14A, C20A and C24A, respectively. Subsequently, all the four cysteine residues, which are common in most of the plant defensins, at positions 3, 14, 20 and 24 were mutated to alanine and the quadruple mutant was named as mutfour. The idea of doing this quadruple mutant is to study the role of the additional fifth disulfide bond (which is a characteristic feature) in PhD1. In addition, the residues forming the five disulfide bonds were mutated to alanine in the native structure and named as mutfive.

\subsection{Molecular Dynamics Simulation}

Energy minimization and simulations were performed using GROMACS v.3.3 [21] with the OPLS-AA/L all-atom force field [22, 23]. Energy minimization was performed using the conjugate-gradient method for $200 \mathrm{ps}$ with the maximum force field cutoff being $1 \mathrm{KJ} \mathrm{mol}^{1}$. The protein models were solvated with the SPC (simple point charge) water model using the genbox program available in the GROMACS suite. The box type used was cubic with the size of $4.8 \times 4.8 \times 4.8 \mathrm{~nm}$. Chloride ions were used to neutralize the overall charge of the system. Parrinello-Rahman protocol was used for pressure and Nose-Hoover coupling protocol was used for temperature. Long range electrostatics was

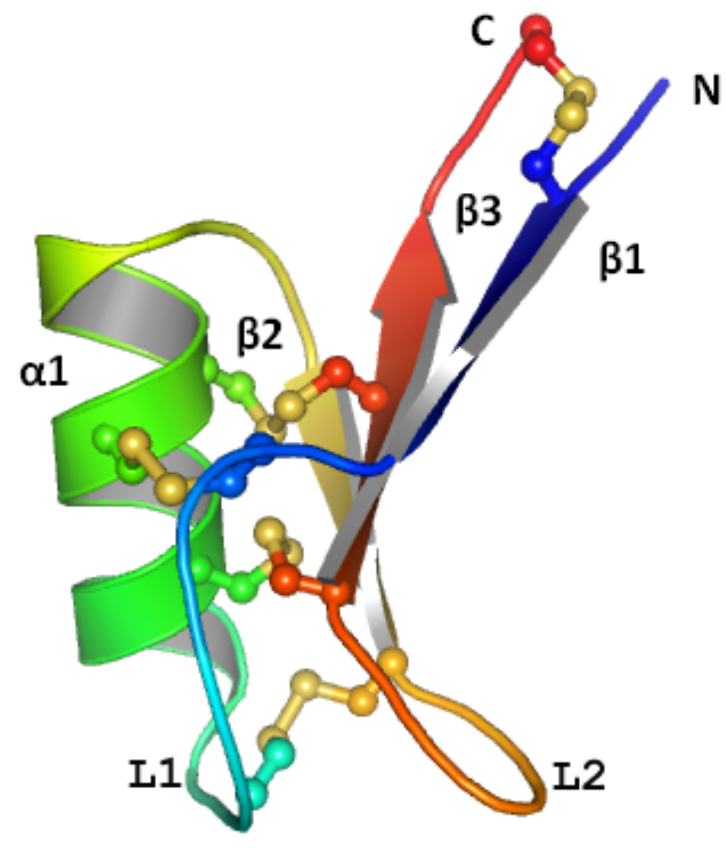

Fig. (1). Schematic diagram of the crystal structure of PhD1, used as the initial structure in all MD simulations. The $\alpha$-helix is shown in green color and the three $\beta$-strands are shown in dark blue, yellow and red respectively. The loop regions (L1 and L2) are colored in cyan and orange, respectively. All the five disulfide bonds are shown in ball and stick representation. The program PYMOL [27] is used to generate the figure. 
computed using the Particle Mesh Ewald (PME) method [24] and Lennard-Jones energies were cut off at $1.0 \mathrm{~nm}$. Bond lengths were constrained with the LINCS algorithm [25]. Simulations were performed on the native structure at different temperatures $(0 \mathrm{~K}, 100 \mathrm{~K}, 200 \mathrm{~K}, 300 \mathrm{~K}, 400 \mathrm{~K}$ and 490 $\mathrm{K})$ and, however, only one temperature $(300 \mathrm{~K})$ was used for all seven mutant structures. Analyses were performed with tools available in the GROMACS suite. The conformational dynamics of the generated trajectories were analyzed by computing root mean square deviation (RMSD). The root mean square fluctuation (RMSF) was also computed for each amino acid residue to analyze the fluctuation pattern within the peptide. The program GRACE was used for the visualization of the trajectories [26] and the threedimensional structures were visualized using PyMOL [27] and 3 dss [28].

\section{RESULTS}

\subsection{Structure Stability of PhD1}

The structural stability of PhD1 is analyzed using the ensembles generated from $5 \mathrm{~ns}$ MD simulations performed at $300 \mathrm{~K}$. The time evolution of RMSD of the MD trajectory is computed. The RMSD increased rapidly for both the main chain and side chain atoms and the increase is almost twofold in the side chain atoms compared to the main chain deviation (Fig. 2a). The RMSD values obtained upon superposition of the initial and the final MD structures are found to be less than $1.2 \AA$ (Table 1). Analysis of RMSF graph reveals the maximum fluctuation in the residues Asp11, Asn16, Ser35, Ile37 and Leu38 (Fig. 2b). The increased fluctuation of Asp11 can be surmised by the fact that the residue is acidic in nature and its side chain

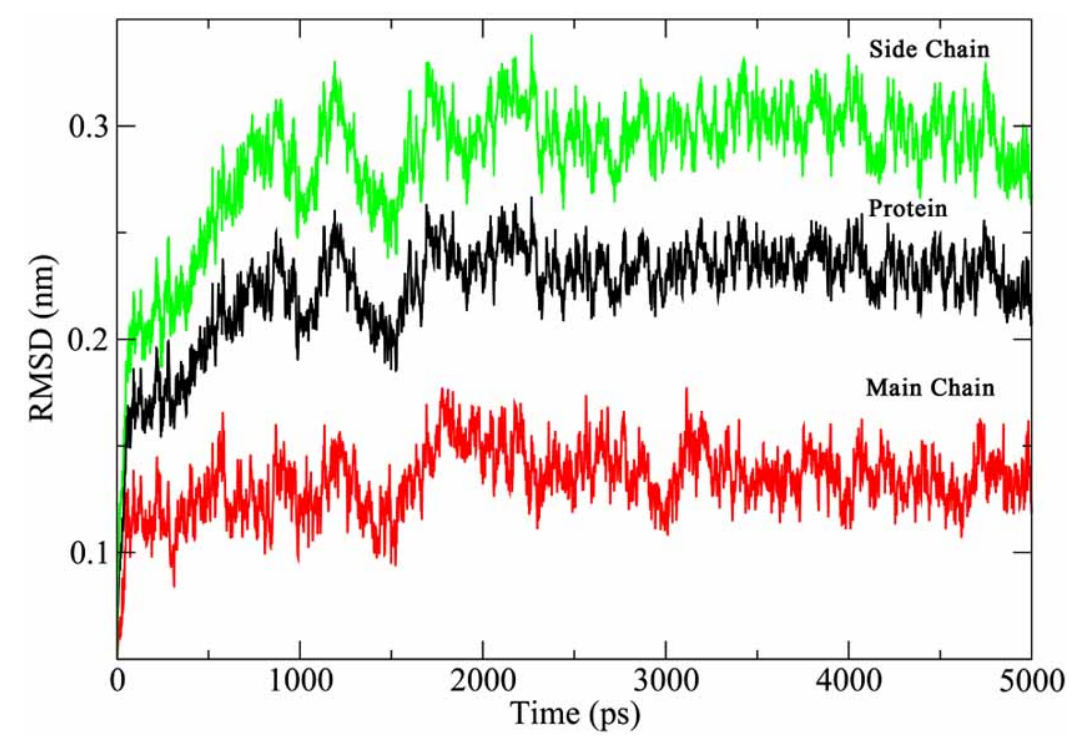

A

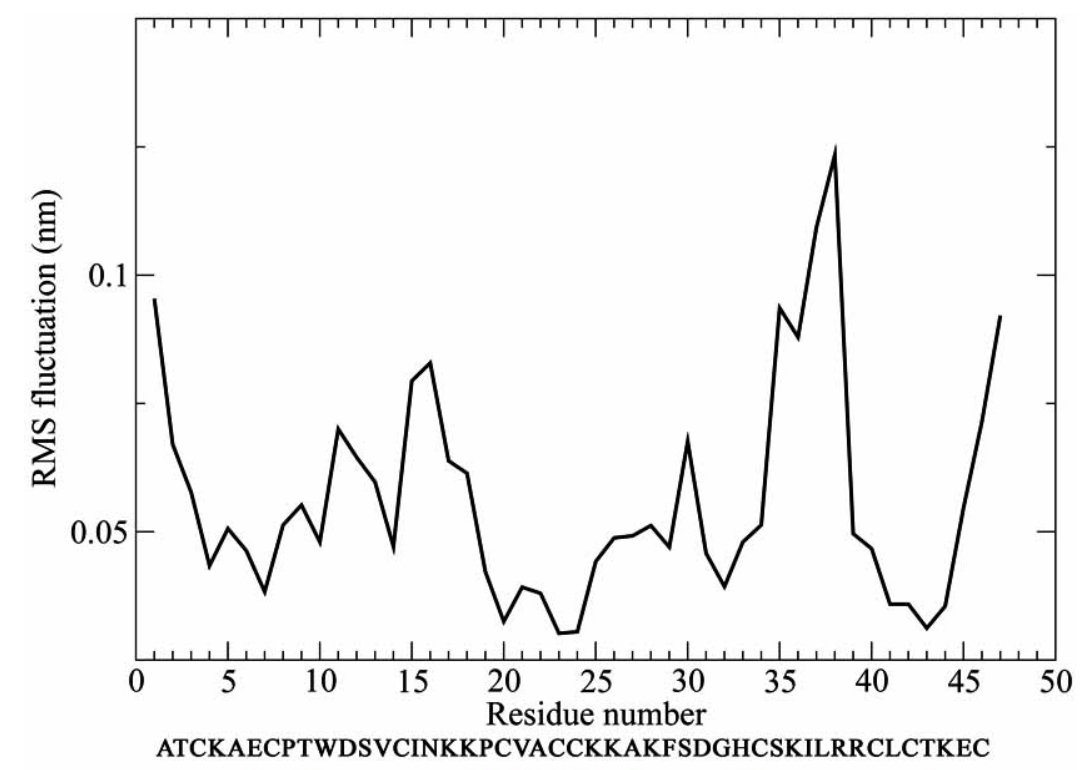

B

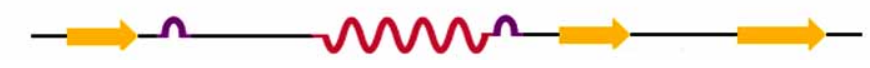

Fig. (2). A graph showing the MD simulations of PhD1 at $300 \mathrm{~K}$. (a) Time evolution of (a) RMSD and (b) RMSF between the MD simulated structures and the native crystal structure [PDB-id: $1 \mathrm{~N} 4 \mathrm{~N}]$. 
carboxylyate group has an intrinsic $\mathrm{pK}_{\mathrm{a}}$ value of 3.9 and, as a result, this residue is ionized and very polar under physiological conditions. The residue Asn 16 displays fluctuations as it is located in the region between the loop L1 and the $\alpha$ helix and thus subjected to directed motion. In the case of Ile37 and Leu38, they are aliphatic in nature and tend to interact with each other more favorably than with water oxygen atoms. All ten cysteine residues in the averaged native structure are found to be very stable showing the least fluctuations (Fig. 2b) and the disulfide bonds formed by these residues are intact.

Table 1. Deviations Obtained on Superposition of the Initial Native PhD1 Structure with the Averaged Structure Obtained after 5 ns Simulations of the Native Structure at Different Temperatures

\begin{tabular}{|c|c|}
\hline Temperature (K) & RMSD ( $\mathbf{\AA})$ \\
\hline \hline 0 & 0.855 \\
\hline 100 & 0.487 \\
\hline 200 & 0.569 \\
\hline 300 & 1.080 \\
\hline 400 & 1.253 \\
\hline 490 & 1.354 \\
\hline
\end{tabular}

The residues Asp11 and Asn16 located in the loop L1, which in turn is located between the $\beta_{1}$-strand and an $\alpha$-helix, account for maximum fluctuation among the residues constituting the loop L1. Further, the residues Leu38 followed by Ile37 and Ser35 display maximum fluctuation among the residues found in loop L2. These results show that the residues making up the loops display the maximum fluctuation compared to the rest of the residues found in the structure. The residues forming an $\alpha$-helix and $\beta$-strands show only small fluctuations and this indicates the structural stability of these secondary structures during the course of simulation.

\subsection{Temperature Dependence of Peptide Structure}

The temperature dependence of $\mathrm{PhD} 1$ is analyzed using 5 ns MD simulations performed at various temperatures by studying the time evolution of RMSD of the MD trajectories. The RMSD values of the native structure simulated at $490 \mathrm{~K}$ is the largest followed by trajectories evolved at $400 \mathrm{~K}$ and $300 \mathrm{~K}$ (Fig. 3). The RMSD values for 0,100 and $200 \mathrm{~K}$ is found to be the lowest and did not fluctuate significantly over the simulation time. It is clear from Figs. (3) and (4), the fluctuations for $100 \mathrm{~K}$ is less compared to that of observed at $0 \mathrm{~K}$. The reasons for this behavior are not known. However, a clear trend is observed for other temperatures studied. The RMSF value for each residue at different temperatures studied relates to the contribution of each residue to the peptide flexibility. A maximum fluctuation is observed at the trajectory corresponding to 490 $\mathrm{K}$, which indicates increased mobility of the threedimensional structure. The residues encompassing the $\beta$ strands and an $\alpha$-helix show small RMSF values at all temperatures except at $490 \mathrm{~K}$ (Fig. 4). The comparison of the secondary structures of the MD trajectories at various

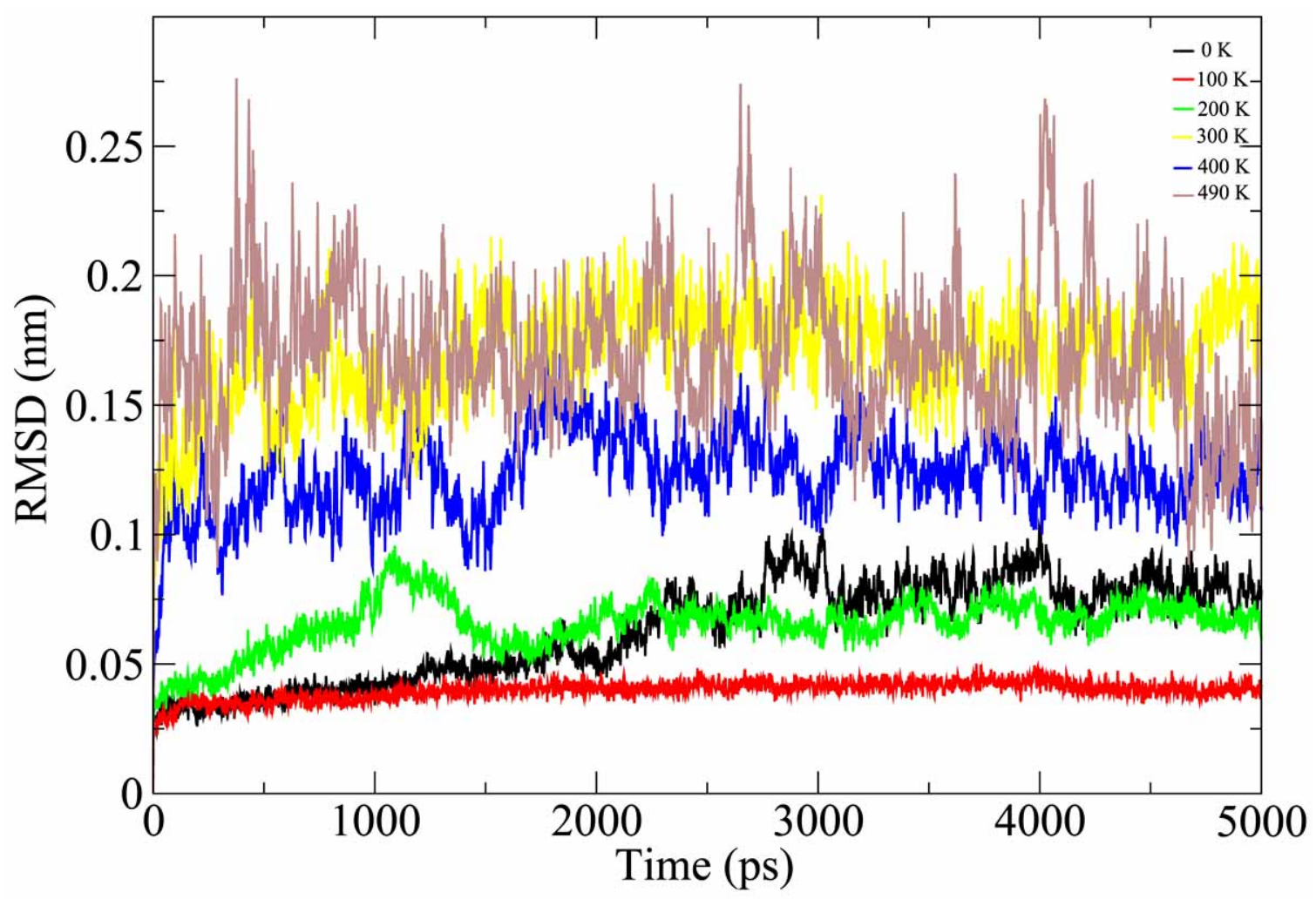

Fig. (3). A graph showing the time evolution of RMSD between the MD simulated structures and the native crystal structure [PDB-id: $1 \mathrm{~N} 4 \mathrm{~N}]$ at different temperatures. 


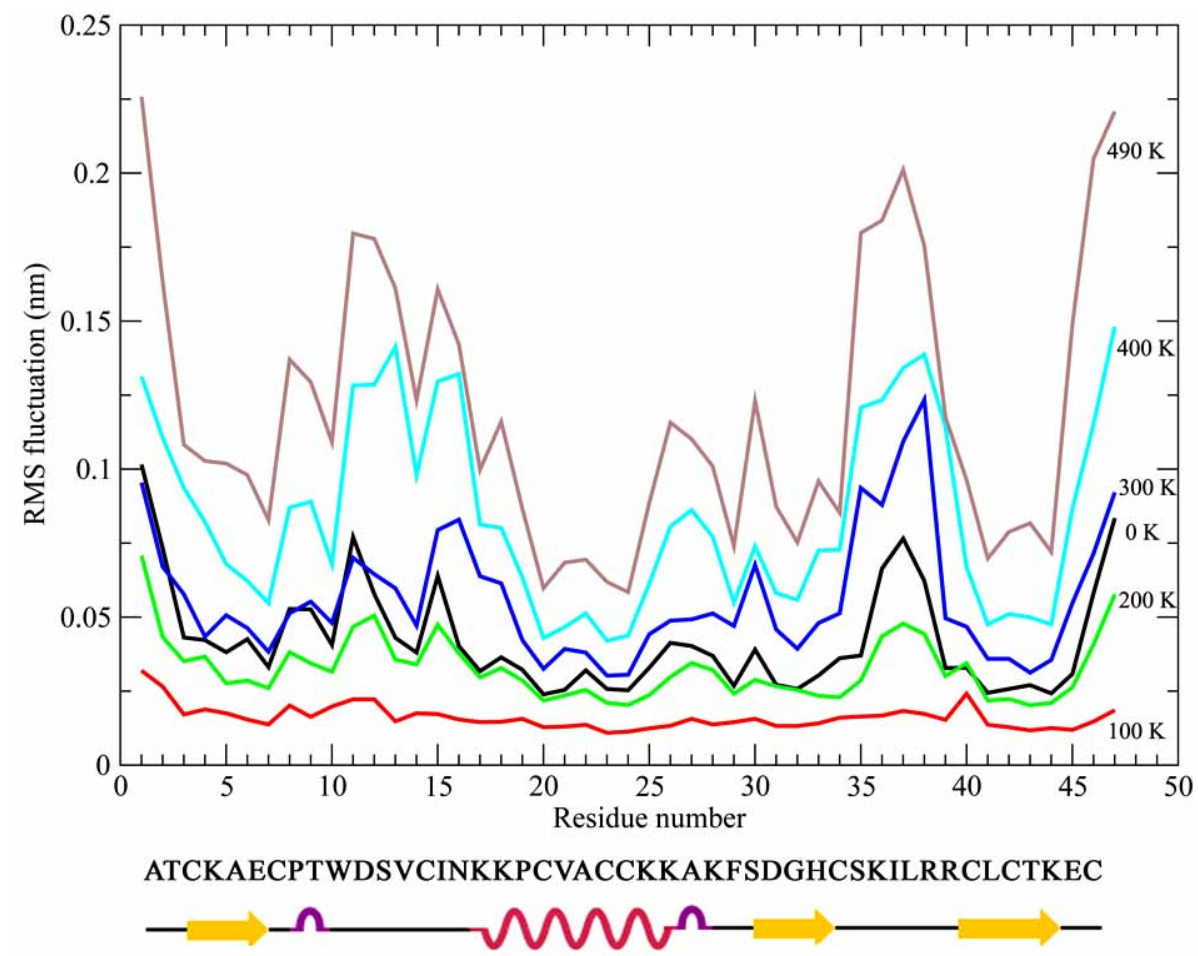

Fig. (4). Effect of temperatures on RMS fluctuations plotted as a function of residue numbers.

temperatures indicates that the stability of the structure slowly decreases with increase in temperature. Among the secondary structures, $\alpha$-helix is preserved across all temperatures while the stability of the $\beta$-strands varies with a change in temperature (Fig. 5).

During the course of simulation, all the cysteine residues aiding in the formation of the disulfide bonds are not affected by change in temperature and are relatively rigid. Significant fluctuations in both the loops (L1 and L2) are observed at $400 \mathrm{~K}$ and $490 \mathrm{~K}$ indicating possible loss of antifungal activity of the peptide, since the residues essential for antifungal activity have been located in the $\alpha$-helix, $\beta_{3^{-}}$ strand, loop L1 (connecting the $\alpha$-helix and $\beta_{1}$-strand) and loop L2 (connecting the strands $\beta_{2}$ and $\beta_{3}$ ) [13]. The L1 loop

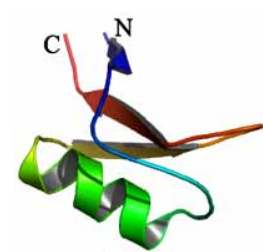

(a)

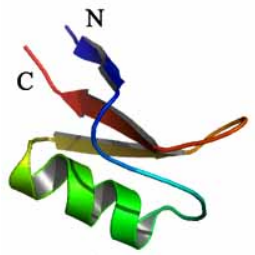

(c)

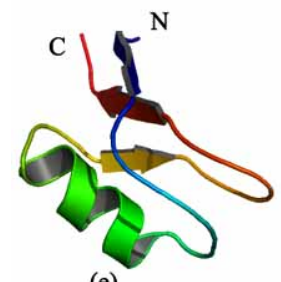

(e)

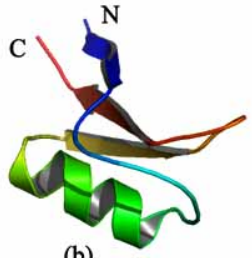

(b)
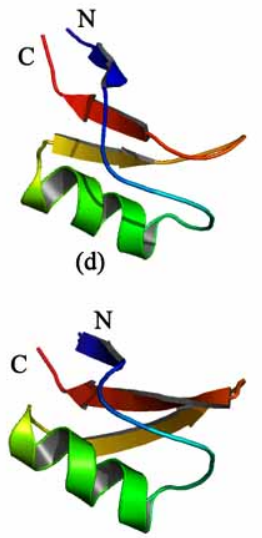

(f)

Fig. (5). Schematic diagrams showing the variation in the three-dimensional structure of PhD1 at different temperatures. Figs. (a), (b), (c), (d), (e) and (f) show cartoon representation of PhD1 at $0 \mathrm{~K}, 100 \mathrm{~K}, 200 \mathrm{~K}, 300 \mathrm{~K}, 400 \mathrm{~K}$ and $490 \mathrm{~K}$, respectively. 
is stabilized by a conserved aromatic residue Trp10 which does not significantly fluctuate, indicating unusual conformational stability. To conclude, the high RMSD and increased flexibility beyond $400 \mathrm{~K}$ suggests significant change in the conformation of the native structure.

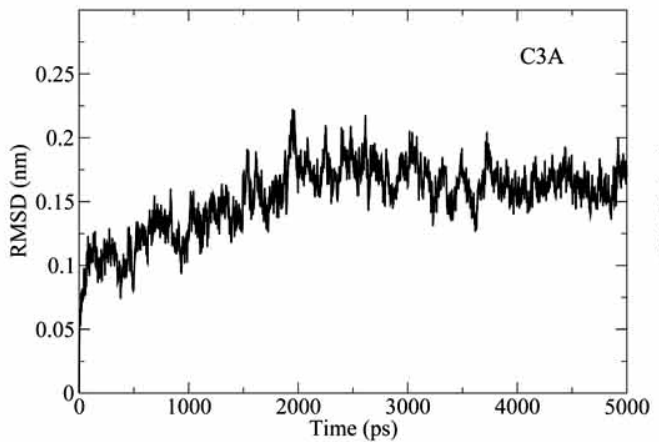

(a)

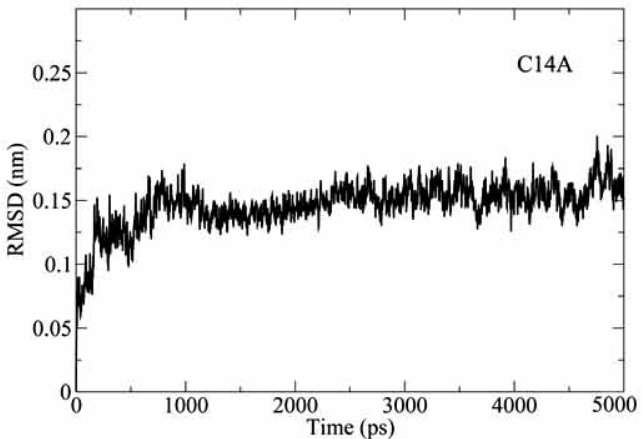

(c)

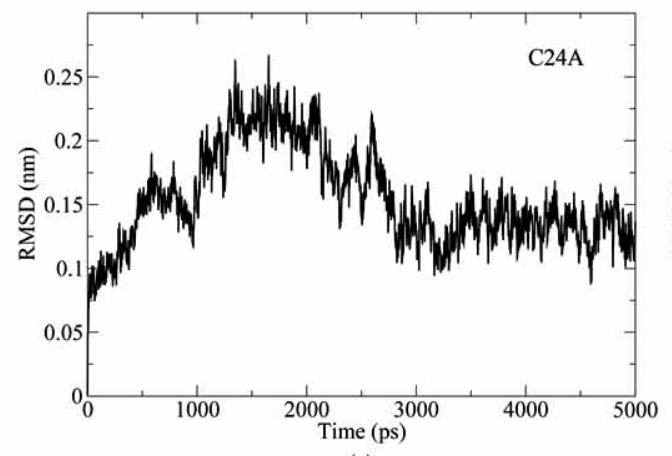

(e)
3.3 Structure and Dynamics of the Mutants: A Comparison with the Native Structure

A $5 \mathrm{~ns}$ MD simulation is performed at $300 \mathrm{~K}$ on the native and the mutant structures. A variation is observed in the RMSDs of the mutant structures (Fig. 6). In most of the

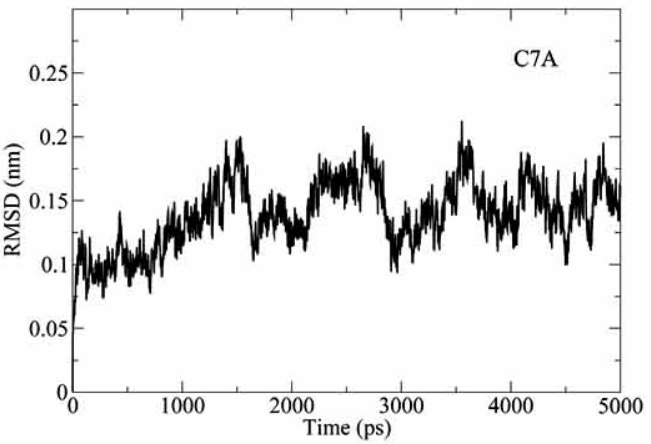

(b)

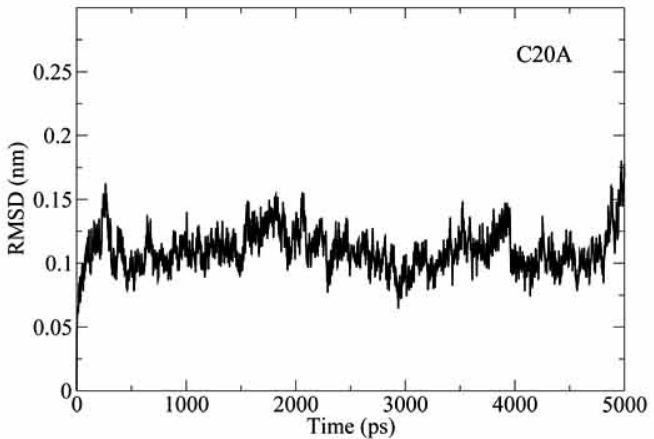

(d)

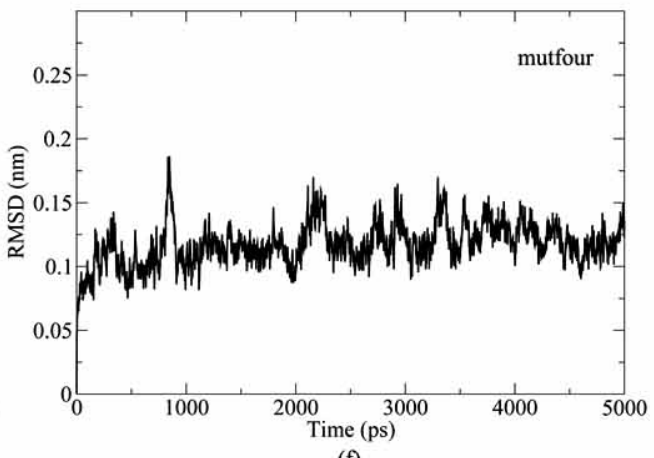

(f)

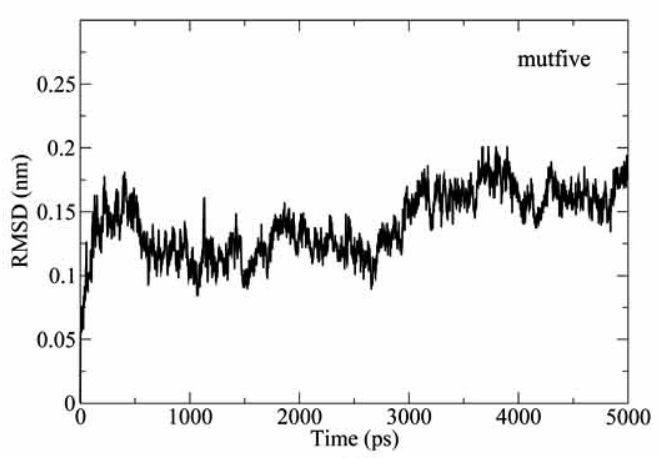

(g)

Fig. (6). A graph showing the RMS deviations of the backbone atoms of all the mutant structures as a function of simulation time. Figs. (a), (b), (c), (d), (e), (f) and (g) represent deviations obtained in all the seven mutants (C3A, C7A, C14A, C20A, C24A, mutfour and mutfive), respectively. The averaged structures are calculated at the end of 5 ns simulations. 
mutant structures, the variation increased within 500 ps and then begins to fluctuate during the course of simulation. The drift in the deviation observed in the mutant structures may be due to the fact that they are as a result of structural changes upon mutation. The results obtained from the superposition of the native and the mutant structures confirmed that the maximum deviation is found to be associated with the mutfive structure (Table 2) in which residues forming the five disulfide bonds are mutated. It is noteworthy that, of all the single mutants analyzed, the maximum RMS deviation is found to be associated with C7A structure, which elucidates the role played by the fifth disulfide bond in rendering increased conformational stability. The fifth bond also plays an important role in stabilizing the loop region between the $\alpha$-helix and $\beta_{1}$-strand and reinforces the hydrogen bond between Thr9 and Lys 26 [13]. In other plant defensins, the positions corresponding to Cys7 and Cys23 are occupied by a serine and a hydrophobic/aromatic residue, respectively. In $\mathrm{PhD} 1$ structure, the presence of the cysteine residues at positions 7

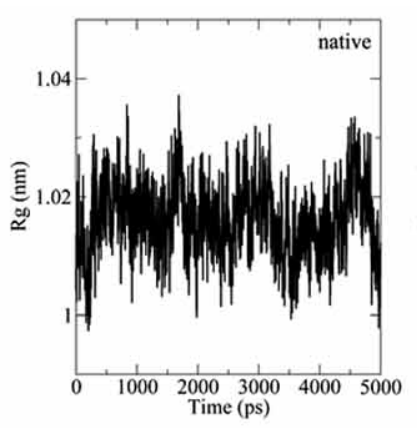

(a)

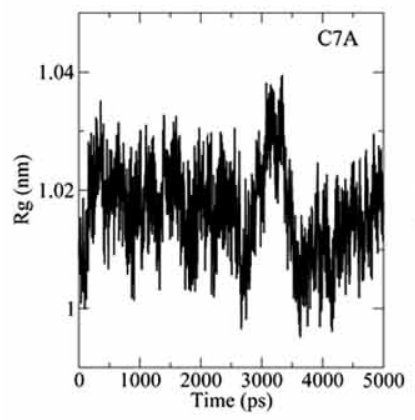

(c)

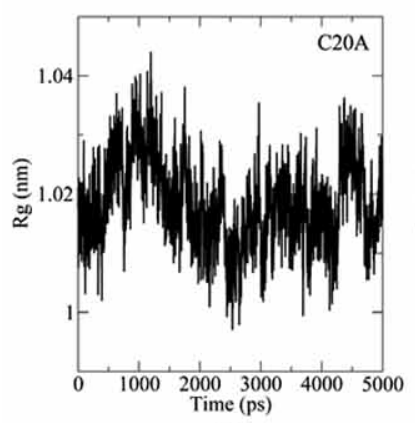

(e)

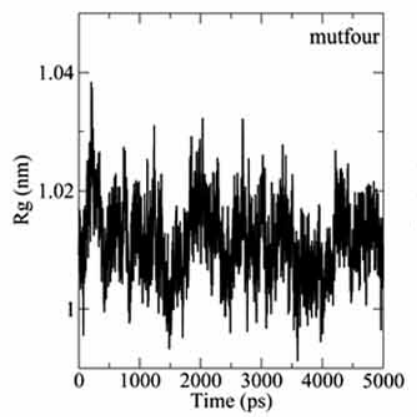

(g)

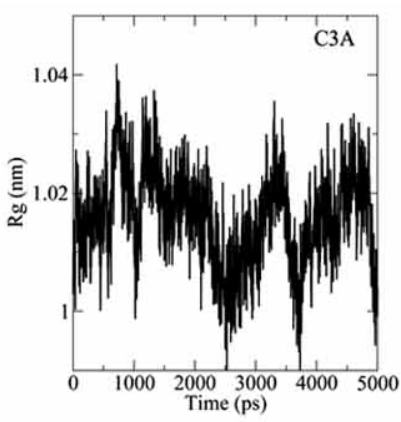

(b)

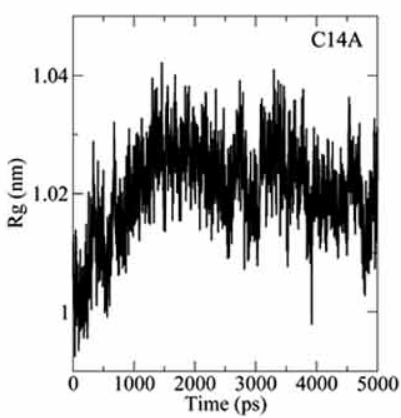

(d)

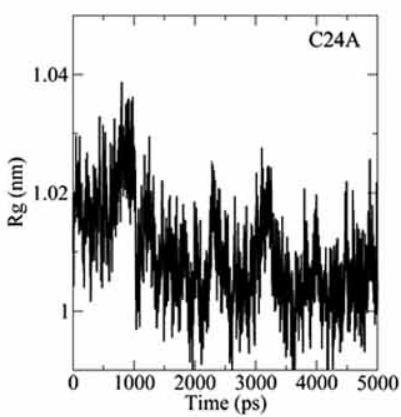

(f)

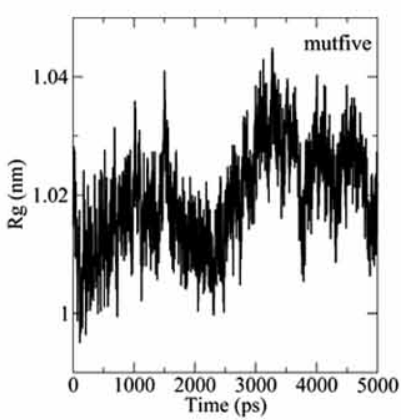

(h)

Fig. (7). $R_{g}$ values of the native and mutant structures plotted as a function of simulation time. Figs. (a), (b), (c), (d), (e), (f), (g) and (h) show fluctuations associated with the native, five single mutants, the quadruple mutant (mutfour) and mutfive structures, respectively. 
Table 2. Deviations Obtained on Superposition of the Native Structure of PhD1 with the Averaged Structure of the Mutants Obtained after 5 ns Simulations

\begin{tabular}{|c|c|}
\hline Mutant Structures & RMSD (̊̊) \\
\hline \hline C3A & 1.196 \\
\hline C7A & 1.321 \\
\hline C14A & 0.908 \\
\hline C20A & 1.298 \\
\hline C24A & 0.987 \\
\hline mutfour & 1.416 \\
\hline mutfive & 1.536 \\
\hline
\end{tabular}

and 23 result in formation of a disulfide bond. Hence, the replacement of hydrophobic interactions with a disulfide bond results in conferring increased stability. Further, radius of gyration $\left(R_{g}\right)$, a parameter indicating structural volume, is

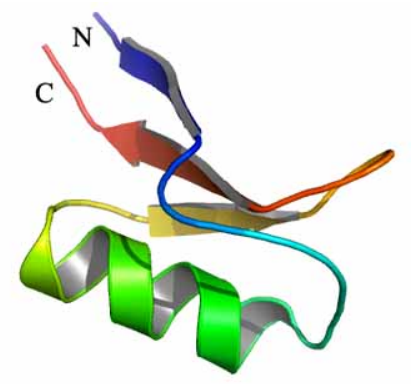

(a)

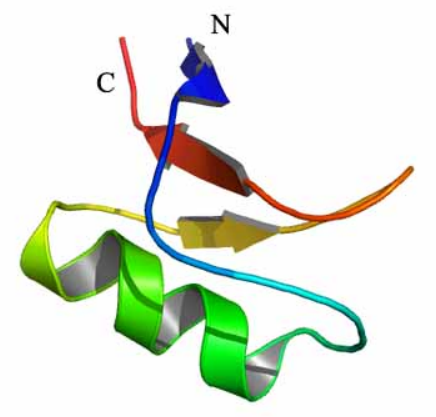

(c)

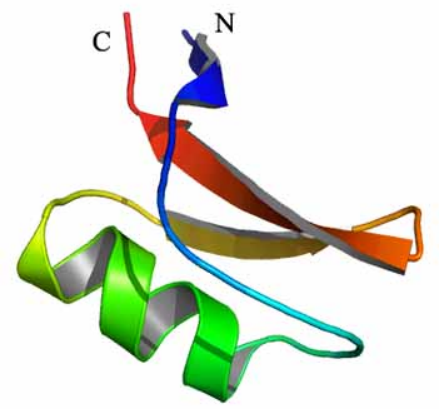

(e) also computed and analyzed. The $\mathrm{R}_{\mathrm{g}}$ values of the native and the mutant structures are plotted as a function of simulation time (Fig. 7). No large variation in $R_{g}$ values is observed between the native and the mutant structures, indicating high structural similarity. Fig. (8) shows the cartoon representation of the native and the five single mutant structures (a: native, b: C3A, c: C7A; d: C14A, e: C20A, f: C24A). It is clear that, the number of residues involved in the secondary structures is less in the single mutant structures, compared to the native structure (Fig. 8). For example, a decrease in $\alpha$ helix content is seen in the $\mathrm{C} 14 \mathrm{~A}$ and $\mathrm{C} 24 \mathrm{~A}$ structures, whereas, in the $\mathrm{C} 7 \mathrm{~A}, \mathrm{C} 14 \mathrm{~A}$ and $\mathrm{C} 20 \mathrm{~A}$ structures, the $\beta$ sheet conformation is slightly altered. Thus, it is apparent that the disulfide bonds play a role in preserving the secondary structural elements.

\section{DISCUSSION}

The dynamics of the native structure performed for $5 \mathrm{~ns}$ at $300 \mathrm{~K}$ shows that it is stable throughout the simulation with all the secondary structure elements preserved in their

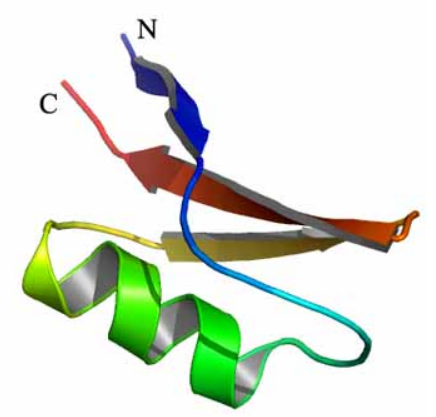

(b)

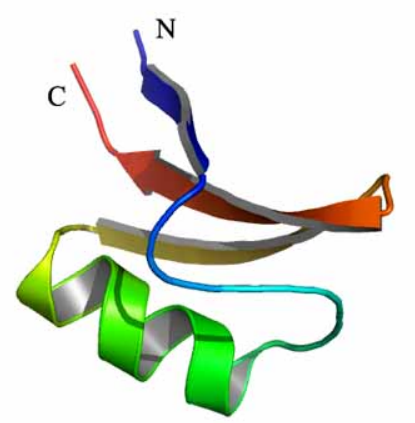

(d)

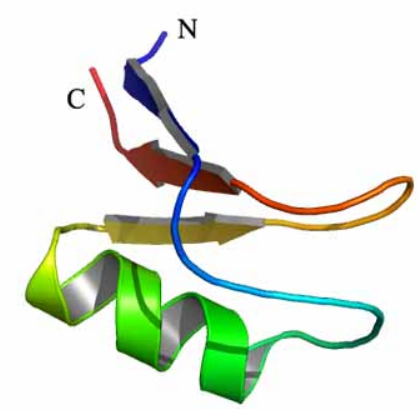

(f)

Fig. (8). Figs. (a), (b), (c), (d) (e) and (f) show cartoon representation of the native and single mutant C3A, C7A, C14A, C20A and C24A structures, respectively. These diagrams show the changes in the secondary structures ( $\alpha$-helix and $\beta$-strands) of PhD1. 
respective orientations and no large deviations are observed. Analysis of RMSF shows that the maximum fluctuation is found to be associated with the residues forming the loop regions (L1 and L2). The result is in good agreement with the experimental observation showing that loops are more flexible than helix and strands [29]. The results of simulations performed at different temperatures indicate that the native structure $\mathrm{PhD} 1$ is stable (except at $490 \mathrm{~K}$ ) and all the secondary structures are intact at all temperatures used in the calculation. A similar trend is also observed in the thermostable antifungal peptide of Aspergilus clavatus [30]. This result also corroborates with the experimental data showing that high temperatures are more detrimental to a protein structure than ambient and lower temperatures [31, 32]. The $\beta$-strands are found to be stable and significant fluctuations are observed only at the maximum temperature $(490 \mathrm{~K})$, which supports experimental results that $\beta$-sheets posses high intrinsic stability [33]. However, the $\alpha$-helix is unusually stable at all temperatures used in the molecular dynamics simulations. A similar high thermal stability of an $\alpha$-helix is also observed in the bacterial Trwc protein [34].

The MD simulations of the mutant structures highlight the importance of additional fifth disulfide bond formed between the cysteine residues Cys7 and Cys23. However, the study of mutfour structure does not yield any conclusive result that one may attribute to the additional fifth disulfide bond. It is reported that the extra disulfide bond restricts the variability of the loop L1 and thus further enhancing structural stablility of PhD1 [13]. The above results show that the fifth additional disulfide bond plays an important role in conferring increased stability to the PhD1 structure. Thus, results obtained from the study of C7A structure throws light on the effect of fifth disulfide bond on the tertiary structure. Further, in the single mutant structures, a small conformational change is observed in $\alpha$-helix and $\beta$ strands (Fig. 8). The RMSD values obtained when all five disulfide bonds are mutated (mutfive) is found to be less than $1.6 \AA$ indicating that the loss of the disulfide bonds did not result in a large conformational change. The $\mathrm{R}_{\mathrm{g}}$ plot of the native and the seven mutant structures is found to be congruent and indicates the structural similarity between the native and the mutant structures. These results are in good agreement with the earlier conclusions in the case of antimicrobial peptides gomesin and protegrin [35].

\section{CONCLUSION}

The floral defensin PhD1 is found to be stable up to 400 $\mathrm{K}$ and shows that the structure is temperature resistant. The present molecular dynamics simulations study on the native and the mutant structures reveals the significance of the additional fifth disulfide bond. It is found to render higher conformational stability, compared to other disulfide bonds, which are generally found in plant defensins. Small conformational variations are observed in the secondary structural elements of the single mutant structures. The consequences of these conformational changes on the antifungal activity should further be experimentally investigated. This study also reveals that even when the residues involved in all five disulfide bonds are mutated, the conformation of the tertiary structure is not significantly altered. The antifungal activity of $\mathrm{PhD1}$ coupled with high structural stability, which is elucidated by our research, makes it an attractive choice for the development of novel fungicides. However, further studies are needed to throw light on the other salient features of the additional disulfide bond.

\section{ACKNOWLEDGEMENTS}

The work reported in the manuscript is fully supported by the Department of Biotechnology (DBT), Government of India. The authors gratefully acknowledge the facilities at the Supercomputer Education and Research Centre, the Interactive Graphics Based Molecular Modelling (IGBMM) and Distributed Information Centre (DIC).

\section{REFERENCES}

[1] Selitrennikoff CP. Antifungal Proteins. Appl Environ Microbiol 2001; 67: 2883-94.

[2] Xiang Y, Huang RH, Liu XZ, Zhang Y, Wang DC. Crystal structure of novel antifungal protein distinct with five disulfide bridges from Eucommia ulmoides Oliver at an atomic resolution. $\mathbf{J}$ Struct Biol 2004; 148: 86-97.

[3] Broekaert WF, Cammue BPA, De Bolle MFC, Thevissen K, De Samblanx GW, Osborn RW. Antimicrobial peptides from plants. Crit Rev Plant Sci 1997; 16: 267-323.

[4] Zasloff M. Antimicrobial peptides of multicellular organisms. Nature 2002; 415: 389-95.

[5] Orru S, Scaloni A, Giannattasio M, Urech K, Pucci P, Schaller G. Amino acid sequence, S-S bridge arrangement and distribution in plant tissues of thionins from Viscum album. J Biol Chem 1997; 378: 989-96.

[6] Segura A, Morreno A, Garcia-Olmedo F. Novel defensin subfamily from spinach (Spinacia oleracea). FEBS Lett 1998; 435: 159-62.

[7] Fant F, Vranken WF, Broekaert W, Borremans F. Determination of the three-dimensional solution structure of raphanus sativus antifungal protein 1 by ${ }^{1}$ H NMR. J Mol Biol 1998; 279: 257-70.

[8] Martins JC, Maes D, Loris R, et al. H NMR study of the solution structure of Ac-AMP2, a sugar binding antimicrobial protein isolated from Amaranthus caudatus. J Mol Biol 1996; 258: 322-33.

[9] Gao GH, Liu W, Dai JK, et al. Solution structure of PAFP-S: a new knottin-type antifungal peptides from the seeds of Phytolacca americana. Biochemistry 2001; 40: 10973-8.

[10] Lay FT, Schirra HJ, Scanlon MJ, Anderson MA, Craik DJ. The three-dimensional solution structure of $\mathrm{NaD} 1$, a new floral defensin from Nicotiana alata and its application to a homology model of the crop defense protein alfAFP. J Mol Biol 2003; 325: 175-88.

[11] Broekaert WF, Terras FR, Cammue BP, Osborn RW. Plant defensins: novel antimicrobial peptides as components of the host defense system. Plant Physiol 1995; 108: 1353-8.

[12] Cornet B, Bonmatin JM, Hetru C, Hoffmann JA, Ptak M, Vovelle F. Refined three-dimensional solution structure of insect defensin A. Structure $1995 ; 3: 435-48$.

[13] Janssen BJC, Schirra HJ, Lay FT, Anderson MA, Craik DJ. Structure of petunia hybrida defensin 1, a novel plant defensin with five disulfide bonds. Biochemistry 2003; 42: 8214-22.

[14] Jerome G, Le-Nguyen D, Gelly JC, Kass Q, Heitz A, Chiche L. KNOTTIN: the knottin or inhibitor cystine knot scaffold in 2007. Nucleic Acid Res 2008; 36: D314-319.

[15] Gelly JC, Gracy J, Kass Q, Le-Nguyen D, Heitz A, Chiche L. The KNOTTIN website and database: a new information system dedicated to the knottin scaffold. Nucleic Acid Res 2004; 32: D156-9.

[16] Thevissen K, Ghazi A, De Samblanx GW, Brownlee C, Osborn RW, Brokaert WF. Fungal membrane responses induced by plant defensins and thionins. J Biol Chem 1996; 271: 15018-25.

[17] DeSamblanx GW, Goderis IJ, Thevissen K, et al. Mutational analysis of a plant defensin from radish (Raphanus sativus L.) reveals two adjacent sites important for antifungal activity. J Biol Chem 1997; 272: 1171-9.

[18] Lay FT, Brugliera F, Anderson MA. Isolation and properties of floral defensins from ornamental tobacco and petunai. Plant Physiol 2003; 131: 1283-93. 
[19] Aliste M, MacCallum J, Tieleman D. Molecular dynamics simulations of pentapeptides at interfaces: salt bridge and cation- $\pi$ interactions. Biochemistry 2003; 42: 8976-87.

[20] Guex N, Peitsch MC. Swiss-model and the Swiss-PdbViewer: An environment for comparative protein modeling. Electrophoresis 1997; 18: 2714-23.

[21] Spoel DVD, Lindahl E, Hess B, Groenhof G, Mark AE, Berendsen HJC. GROMACS: Fast, flexible, and free. J Comput Chem 2005; 26: 1701-18.

[22] Jorgensen WL, Maxwell DS, Tirado-Rives J. Development and testing of the OPLS all-atom force field on conformational energetics and properties of organic liquids. J Am Chem Soc 1996; 1225-36.

[23] Kaminski GA, Friesner RA, Tiraso-Rives J, Jorgensen WL. Evaluation and reparametrization of the OPLS-AA force field for proteins via comparison with accurate quantum chemical calculations on peptides. J Phys Chem 2001; 105: 6474-87.

[24] Darden TD, York PL. Particle mesh Ewald: An $N \cdot \log (N)$ method for Ewald sums in large systems. J Chem Phys 1993; 98: 10089-92.

[25] Hess B, Bekker H, Berendsen HJC, et al. LINCS: A linear constraint solver for molecular simulations. J Comput Chem 1997; 18: $11225-36$.

[26] Grace [homepage on the Internet]. C1998-2000 [cited 2008 Oct] Available from: http://plasma-gate.weizmann.ac.il/Grace/

[27] Delano WL. The PyMOL molecular graphics system, Delano Scientific, Palo Alto, CA, USA, 2002.
[28] Sumathi K, Ananthalakshmi P, Roshan MN, Sekar K. 3dss: 3dimensional structural superposition. Nucleic Acids Res 2006; 34: W128-34.

[29] Nemoto T, Uebayasi M, Komeiji Y. Flexibility of a loop in a pheromone binding protein from Bombyx mori: a molecular dynamics simulation. Chem-Bio Informatics J 2002; 2: 32-7.

[30] Gargouri HS, Gargouri A. First isolation of a novel thermostable antifungal peptide secreted by Aspergillus clavatus. Peptides 2008; 29: $1871-7$.

[31] Smeller L. Pressure-temperature phase diagrams of biomolecules. Biochim Biophys Acta 2002; 1595: 11-29.

[32] Xu Y, Oyola R, Gai F. Infrared study of the stability and folding kinetics of a 15-residue beta-hairpin. J Am Chem Soc 2003; 125: 1049-74.

[33] Perczel A, Jakli I, Csizmadia I. Intrinsically stable secondary structure elements of proteins: a comprehensive study of folding units of proteins by computation and by analysis of data determined by X-ray crystallography. Chemistry 2003; 9: 5332-42.

[34] Arrondo JLR, Echabe I, Iloro I, Hernando MA, Cruz F, Goni FM. A bacterial TrwC relaxase domain contains a thermally stable alpha-helical core. J Mol Model 2003; 8: 327-35.

[35] Castro JRM, Fuzo CA, Degreve L, Caliri A. The role of disulfide bridges in the 3-D structures of antimicrobial peptides gomesin and protegrin-1: a molecular dynamics study. Genet Mol Res 2008; 7: 1070-88

(C) Jayanthi et al.; Licensee Bentham Open.

This is an open access article licensed under the terms of the Creative Commons Attribution Non-Commercial License (http://creativecommons.org/licenses/by$\mathrm{nc} / 3.0 /$ ), which permits unrestricted, non-commercial use, distribution and reproduction in any medium, provided the work is properly cited. 